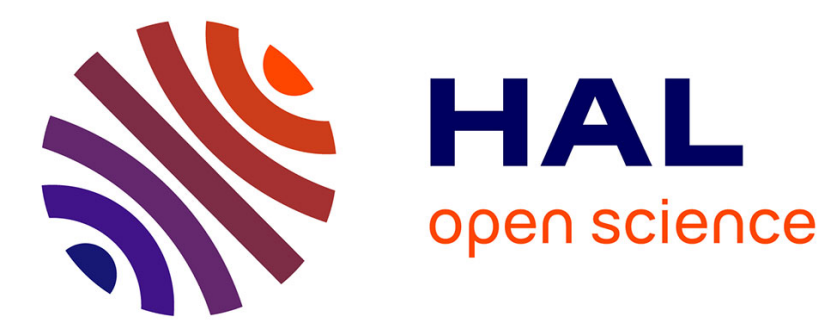

\title{
From cmc surfaces to Hamiltonian Stationary Lagrangian Surfaces
}

\author{
Frédéric Hélein, Pascal Romon
}

\section{To cite this version:}

Frédéric Hélein, Pascal Romon. From cmc surfaces to Hamiltonian Stationary Lagrangian Surfaces. Mathematical Society of Japan 9th International Research Institute on Integrable Systems in Differential Geometry, 2002, Tokyo, Japan. pp.163-187. hal-00733014

\section{HAL Id: hal-00733014 https://hal.science/hal-00733014}

Submitted on 17 Sep 2012

HAL is a multi-disciplinary open access archive for the deposit and dissemination of scientific research documents, whether they are published or not. The documents may come from teaching and research institutions in France or abroad, or from public or private research centers.
L'archive ouverte pluridisciplinaire HAL, est destinée au dépôt et à la diffusion de documents scientifiques de niveau recherche, publiés ou non, émanant des établissements d'enseignement et de recherche français ou étrangers, des laboratoires publics ou privés. 
Advanced Studies in Pure Mathematics 99, 2006

Surveys on Geometry and Integrable Systems

pp. $0-0$

\title{
From CMC surfaces to Hamiltonian stationary Lagrangian surfaces
}

\author{
Frédéric Hélein and Pascal Romon
}

\section{$\S 1$. Introduction}

Minimal surfaces and surfaces with constant mean curvature (CMC) have fascinated differential geometers for over two centuries. Indeed these surfaces are solutions to variational problems whose formulation is elegant, modelling physical situations involving soap films and bubbles; however their richness has not been exhausted yet. Advances in the understanding of these surfaces draw on complex analysis, theory of Riemann surfaces, topology, nonlinear elliptic PDE theory and geometric measure theory. Furthermore, one of the most spectacular developments in the past twenty years has been the discovery that many problems in differential geometry - including those of minimal and CMC surfaces are actually integrable systems.

The theory of integrable systems developed in the 1960's, beginning essentially with the study of a now famous example: the Korteweg-de Vries equation, $u_{t}+6 u u_{x}+u_{x x x}=0$, modelling waves in a shallow flat channel $^{1}$. In the 1960's mathematicians noticed the exceptional properties of the KdV equation: existence of solitary waves that "superpose" almost linearly, and an infinite number of conserved quantities. From these observations, C. Gardner, J. Greene, M. Kruskal and R. Miura [12] showed in 1967 that this equation could be solved completely by reducing it to a linear one, thanks to a rather sophisticated nonlinear transformation called inverse scattering. In 1968, P. Lax [23] gave an

Received December 27, 2000.

Revised February 8, 2002.

${ }^{1}$ Earlier already, in 1955, Fermi, Pasta and Ulam had unexpectedly discovered the soliton phenomenon (to their great surprise) while simulating a one-dimensional model in statistical mechanics on the Los Alamos computer. 
interpretation of their method by noticing that the $\mathrm{KdV}$ equation can be rewritten as the evolutionary equation for an operator $L$ (in fact $L=-\frac{d^{2}}{d x^{2}}+u$ ), namely $L_{t}=[A, L]$. In particular the spectrum of $L$ is time independent, explaining the infinite number of conserved quantities. That was the starting point for a series of increasingly deeper observations on $\mathrm{KdV}$, formulated notably by the Japanese and Russian schools, aiming at interpreting its miraculous properties: Hamiltonian structure, explicit formulation of some of its solutions (called finite gap solutions) through techniques from algebraic geometry, relation with dynamical systems on Lie algebras, and so on... For all these properties the $\mathrm{KdV}$ equation deserves the name of completely integrable system. Later on other equations coming from physics were identified as completely integrable systems: the one dimensional nonlinear Schrödinger, Toda field equation, and so on... Today however differential geometry seems to be the field where most of the completely integrable systems are discovered (see [33] for instance). We will see in the following some examples stemming from problems in Riemannian geometry. Other very interesting examples are linked to geometrical problems (but inspired by physics) in four dimensions: self-dual Yang-Mills connections, and self-dual Einstein metrics (the starting point being Penrose's theory of twistors).

The intrusion of integrable systems in differential geometry may seem a recent discovery, however it is not so, since a "prehistory" of completely integrable systems in geometry occurred one century ago. A number of clues had been spotted by nineteenth century geometers: conjugate families of CMC surfaces discussed by O. Bonnet in 1853 [5], the Enneper-Weierstrass representation for minimal surfaces (see below), construction of constant mean or constant Gaussian curvature surfaces by A. Enneper and his students in 1868 and 1880 [10, 8, 22, 24], construction of soliton-like solutions through iteration of Bäcklund transformations [3, 25]. A synthesis can be found in the book of G. Darboux [7]. Of course the geometers of that period did not have the point of view we have today, which is based on concepts from Lie algebra theory.

In this survey we wish to present this theory in an accessible way, through the examples of minimal surfaces, CMC surfaces and harmonic maps or Willmore surfaces. We will then present a more recent example, discovered by both authors $[17,18,19]$. 


\section{§2. Minimal surfaces and CMC surfaces}

Let $m$ be a point of an oriented surface $\Sigma \subset \mathbb{R}^{3}$. We define the principal curvatures at $m$ as follows. Consider the one-parameter family of affine planes $P$ through $m$, perpendicular to the tangent plane to $\Sigma$ at $m, T_{m} \Sigma$. Any such plane $P$ cuts $\Sigma$ along a (planar) curve and we let $k(P)$ be the (oriented) curvature of that curve at $m$, obviously dependent on $P$. As $P$ revolves around the normal line at $m, k(P)$ oscillates; its minimal and maximal values are called principal curvatures and denoted by $k_{1} \leq k_{2}$. If $k(P)$ is independent of $P$, i.e. $k_{1}=k_{2}$, the point $m$ is called umbilic. The quantity $H:=\frac{1}{2}\left(k_{1}+k_{2}\right)$ is the mean curvature of $\Sigma$ at $m$ and the product $K=k_{1} k_{2}$ is the Gaussian curvature.

A minimal surface satisfies by definition $H=0$ at all points. The reason behind the name is that such surfaces are stationary with respect to the area functional $\mathcal{A}(\Sigma)=\int_{\Sigma} d a$ (not necessarily minimizing ${ }^{2}$ ). A very simple experimental procedure for producing minimal surfaces consists of dipping a closed metal wire in soap and water: when taken out of the liquid, the wire bounds a soap film, materializing a minimal surface.

Constant mean curvature surfaces are by convention those surfaces with constant non-zero mean curvature $H$, as opposed to minimal surfaces.

Using conformal coordinates turns out to be a very convenient way of studying the properties of these surfaces. Indeed, for any simply connected immersed surface $\Sigma$ there exists a conformal parametrization $X: \Omega \rightarrow \mathbb{R}^{3}$, where $\Omega$ is an open subset of $\mathbb{C}$, i.e.

$$
\left|\partial_{x} X\right|^{2}-\left|\partial_{y} X\right|^{2}-2 i\left\langle\partial_{x} X, \partial_{y} X\right\rangle=0,
$$

and $X(\Omega)=\Sigma$. Let $u$ map $z=x+i y \in \Omega$ to the oriented unit normal vector at $X(z)$ :

$$
u(z):=\frac{\partial_{x} X \times \partial_{y} X}{\left|\partial_{x} X \times \partial_{y} X\right|},
$$

where $\times$ denotes the cross product in $\mathbb{R}^{3}$. The map $u$ is called the Gauss map. Then the conformality assumption implies that the first and second fundamental forms take the following form:

$$
I:=\left(\begin{array}{cc}
\left|\partial_{x} X\right|^{2} & \left\langle\partial_{x} X, \partial_{y} X\right\rangle \\
\left\langle\partial_{x} X, \partial_{y} X\right\rangle & \left|\partial_{y} X\right|^{2}
\end{array}\right)=e^{2 \omega}\left(\begin{array}{cc}
1 & 0 \\
0 & 1
\end{array}\right)
$$

\footnotetext{
${ }^{2}$ However stationary surfaces will always be minimizing for small perturbations in a small enough compact subset.
} 
and

$$
I I:=\left(\begin{array}{cc}
\left\langle\left(\partial_{x}\right)^{2} X, u\right\rangle & \left\langle\partial_{x} \partial_{y} X, u\right\rangle \\
\left\langle\partial_{x} \partial_{y} X, u\right\rangle & \left\langle\left(\partial_{y}\right)^{2} X, u\right\rangle
\end{array}\right)=e^{2 \omega}\left(\begin{array}{cc}
H+a & b \\
b & H-a
\end{array}\right)
$$

with $\omega, H, a, b$ are real-valued functions. $H$ is exactly the mean curvature. In particular

$$
\Delta X=2 H \partial_{x} X \times \partial_{y} X
$$

Thus all minimal surfaces (respectively, CMC surfaces) are locally described by the conformal immersions $X$ such that $\Delta X=0$ (respectively, $\Delta X=2 H_{0} \partial_{x} X \times \partial_{y} X$ with $H_{0}$ a non-zero constant). As we will see, this way of stating the geometric problem is quite productive.

\subsection{Minimal surfaces}

The set of equations

$$
\left|\partial_{x} X\right|^{2}-\left|\partial_{y} X\right|^{2}-2 i\left\langle\partial_{x} X, \partial_{y} X\right\rangle=0 \quad \text { and } \quad \Delta X=0
$$

may be solved locally by introducing a complex-valued function

$$
f:=\partial_{z} X=\frac{1}{2}\left(\partial_{x} X-i \partial_{y} X\right)
$$

then (3) becomes

$$
f^{2}=0 \quad \text { and } \quad \partial_{\bar{z}} f=0,
$$

since $\partial_{\bar{z}} \partial_{z}=\frac{1}{4} \Delta .\left(f^{2}\right.$ denotes the dot product $\left(f^{1}\right)^{2}+\left(f^{2}\right)^{2}+\left(f^{3}\right)^{2}$. $)$ The solution to these two equations is given by

$$
f=\left(\begin{array}{c}
\frac{i}{2}\left(w^{2}-1\right) \\
\frac{1}{2}\left(w^{2}+1\right) \\
i w
\end{array}\right) h,
$$

where $w$ (respectively, $h$ ) is some meromorphic (respectively, holomorphic) function from $\Omega$ to $\mathbb{C}$. Thus

$$
X(z)=C+\operatorname{Re}\left[\int_{z_{0}}^{z} f(\zeta) d \zeta\right]
$$

with $C$ a constant in $\mathbb{R}^{3}$ and $z_{0}$ a fixed base point in $\Omega$. This is the Enneper-Weierstrass representation formula. 
The reader may observe the following fact: locally, any minimal surface is part of (continuous) one-parameter family of minimal surfaces given by

$$
X_{\lambda}(z)=C+\operatorname{Re}\left[\lambda^{-2} \int_{z_{0}}^{z} f(\zeta) d \zeta\right], \quad \lambda \in S^{1} \subset \mathbb{C}^{\star} .
$$

This family is called the conjugate family ${ }^{3}$ of the surface $\Sigma$.

\subsection{Constant mean curvature surfaces}

Even before Enneper and Weierstrass discovered their representation formula, O. Bonnet [5] found that minimal surfaces and CMC surfaces could be deformed, giving birth to conjugate families. This relies on the fact that the immersion $X$ can be reconstructed with the mere knowledge of the first and second fundamental forms of $X$. More specifically, given four real-valued functions $\omega, H, a, b$, there exists a conformal immersion $X$ whose first and second fundamental forms are given by (1) and (2), under the condition that $\omega, H, a, b$ satisfy some compatibility relations: the Gauss-Codazzi equations ${ }^{4}$. The crucial observation is that $(\omega, H, a, b)$ satisfies the Gauss-Codazzi equations if and only if the deformed data $\left(\omega, H, a_{\lambda}, b_{\lambda}\right)$ does, where

$$
a_{\lambda}-i b_{\lambda}:=\lambda^{-2}(a-i b) \text { for all } \lambda \in \mathbb{C}^{\star} .
$$

Hence starting with a conformal immersion $X$ with constant or zero mean curvature, and deforming the first and second fundamental forms $I$ and $I I$ by substituting $\left(\omega, H, a_{\lambda}, b_{\lambda}\right)$ for $(\omega, H, a, b)$, one gets tensors $I_{\lambda}$ and $I I_{\lambda}$ on $\Omega$ that are the first and second fundamental forms of a new conformal immersion $X_{\lambda}$ of constant or zero mean curvature. One constructs that way the conjugate family of $X$, which coincides with the Enneper-Weierstrass formula (6) in the case of minimal surfaces.

\subsection{Changing viewpoint: the moving frame}

We revisit here the construction of the conjugate family of a constant or zero mean curvature surface. Before doing this, let us make a detour into the theory of moving frames. Consider the projection $p$ from the $X$.

${ }^{3}$ It depends only on the surface, not on the choice of the parametrization

${ }^{4}$ If $H=$ constant, the Gauss-Codazzi equations are: $\frac{\partial}{\partial \bar{z}}\left(e^{2 \omega}(a-i b)\right)=0$ and $\Delta \omega+e^{2 \omega}\left(H^{2}-a^{2}-b^{2}\right)=0$. 
group of affine isometries of $\mathbb{R}^{3}, S O(3) \ltimes \mathbb{R}^{3}$, to $\mathbb{R}^{3}$, defined by $(R, t) \mapsto t$. Recall that $S O(3) \ltimes \mathbb{R}^{3}$ can be identified with the group of four by four real matrices of type $\left(\begin{array}{cc}R & t \\ 0 & 1\end{array}\right)$ with $R \in S O(3)$ and $t \in \mathbb{R}^{3}$. We say that $F$ lifts $X$ if $p \circ F=X$ :

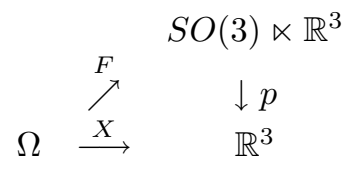

If $\Omega$ is a simply connected domain in $\mathbb{C}$ we can always lift $X$. A moving frame (or Darboux frame) is a lift $F: \Omega \longrightarrow S O(3) \ltimes \mathbb{R}^{3}$ of the following form:

$$
F(z):=\left(\begin{array}{cccc}
e_{1}(z) & e_{2}(z) & u(z) & X(z) \\
0 & 0 & 0 & 1
\end{array}\right)
$$

where, for $z \in \Omega,\left(e_{1}(z), e_{2}(z)\right)$ is any positively oriented orthonormal basis of $T_{u(z)} S^{2} \simeq T_{X(z)} \Sigma$, smoothly varying with $z$. The simplest (but not the only) way to define $\left(e_{1}(z), e_{2}(z)\right)$ is through coordinates: $e_{1}=e^{-\omega} \partial_{x} X, e_{2}=e^{-\omega} \partial_{y} X$, where $e^{2 \omega}:=\left|\partial_{x} X\right|^{2}=\left|\partial_{y} X\right|^{2}$. Notice that the triple $\left(e_{1}, e_{2}, u\right)$ is a positively oriented orthonormal basis of $\mathbb{R}^{3}$ and as such can be identified with an element of $S O(3)$.

As seen above, $X$ is tied to its conjugate family, hence $u$ is tied to the family of Gauss maps $u_{\lambda}$ of $X_{\lambda}$. We set

$$
e_{1, \lambda}-i e_{2, \lambda}:=2 \lambda e^{-\omega} \frac{\partial X_{\lambda}}{\partial z}
$$

obtaining thus a deformation $F_{\lambda}$ of $F$ defined by

$$
F_{\lambda}(z)=\left(\begin{array}{cc}
\phi_{\lambda}(z) & X_{\lambda}(z) \\
0 & 1
\end{array}\right) \text {, where } \phi_{\lambda}=\left(\begin{array}{ccc}
e_{1, \lambda} & e_{2, \lambda} & u_{\lambda}
\end{array}\right) .
$$


Since $F_{\lambda}$ is group-valued, we may compute its Maurer-Cartan form $A_{\lambda}:=F_{\lambda}^{-1} d F_{\lambda}$ and find

$$
\begin{aligned}
A_{\lambda}= & \lambda^{-1}\left(\begin{array}{cccc}
0 & 0 & \left\langle\partial_{z} u, e_{1}\right\rangle & \left\langle\partial_{z} X, e_{1}\right\rangle \\
0 & 0 & \left\langle\partial_{z} u, e_{2}\right\rangle & \left\langle\partial_{z} X, e_{2}\right\rangle \\
-\left\langle\partial_{z} u, e_{1}\right\rangle & -\left\langle\partial_{z} u, e_{2}\right\rangle & 0 & 0 \\
0 & 0 & 0 & 0
\end{array}\right) d z \\
& +\star d \omega\left(\begin{array}{cccc}
0 & -1 & 0 & 0 \\
1 & 0 & 0 & 0 \\
0 & 0 & 0 & 0 \\
0 & 0 & 0 & 0
\end{array}\right) \\
& +\lambda\left(\begin{array}{cccc}
0 & 0 & & \\
0 & 0 & \left\langle\partial_{\bar{z}} u, e_{1}\right\rangle & \left\langle\partial_{\bar{z}} X, e_{2}\right\rangle \\
-\left\langle\partial_{\bar{z}} u, e_{1}\right\rangle & -\left\langle\partial_{\bar{z}} u, e_{2}\right\rangle & 0 & 0 \\
0 & 0 & 0 & 0
\end{array}\right) d \bar{z}
\end{aligned}
$$

Remarkably enough, $A_{\lambda}$ splits into three pieces $\lambda^{-1} A_{1}^{\prime}+A_{0}+\lambda A_{1}^{\prime \prime}$ which can be easily read off from the original Maurer-Cartan form $A=F^{-1} d F$ of $F$. Indeed

$$
F^{-1} d F=\left(\begin{array}{cccc}
0 & -\star d \omega & \left\langle d u, e_{1}\right\rangle & \left\langle d X, e_{1}\right\rangle \\
\star d \omega & 0 & \left\langle d u, e_{2}\right\rangle & \left\langle d X, e_{2}\right\rangle \\
-\left\langle d u, e_{1}\right\rangle & -\left\langle d u, e_{2}\right\rangle & 0 & 0 \\
0 & 0 & 0 & 0
\end{array}\right)
$$

We deduce a more direct way of constructing the conjugate family of $X$, namely start by lifting $X$ to $F: \Omega \longrightarrow S O(3) \ltimes \mathbb{R}^{3}$, split its MaurerCartan form $A$ into two pieces

$$
A_{0}=\star d \omega\left(\begin{array}{cccc}
0 & -1 & 0 & 0 \\
1 & 0 & 0 & 0 \\
0 & 0 & 0 & 0 \\
0 & 0 & 0 & 0
\end{array}\right)
$$

and $A_{1}:=A-A_{0}$, then split again $A_{1}=A_{1}^{\prime}+A_{1}^{\prime \prime}$ where $A_{1}^{\prime}:=A_{1}\left(\frac{\partial}{\partial z}\right) d z$ and $A_{1}^{\prime \prime}=\overline{A_{1}^{\prime}}:=A_{1}\left(\frac{\partial}{\partial \bar{z}}\right) d \bar{z}$. Then the Maurer-Cartan form of $F_{\lambda}$ is just $A_{\lambda}=\lambda^{-1} A_{1}^{\prime}+A_{0}+\lambda A_{1}^{\prime \prime}$. Now given such an $A_{\lambda}$, one may ask whether a corresponding map $F_{\lambda}: \Omega \longrightarrow S O(3) \ltimes \mathbb{R}^{3}$ exists, such that $A_{\lambda}=F_{\lambda}^{-1} d F_{\lambda}$. The answer for a simply connected domain $\Omega$ is positive if and only if

$$
d A_{\lambda}+A_{\lambda} \wedge A_{\lambda}=0 .
$$


However this equation turns out to be exactly equivalent to these two conditions: $A=F^{-1} d F$ and $X$ is a constant or zero mean curvature immersion.

\subsection{Another point of view using the Gauss map}

We have previously defined the Gauss map $u: \Omega \longrightarrow S^{2}$ of an immersion $X$, allowing us later on to characterize the second fundamental form and the lift $F$. There is more to this than one might expect, thanks to the following result.

Theorem 1 (Ruh-Vilms). Let $X$ be a conformal immersion. Then $X$ has constant or zero mean curvature if and only if its Gauss map $u$ is a harmonic map into the unit sphere, i.e. $u$ is a solution of the equation

$$
\Delta u+u|\nabla u|^{2}=0 .
$$

Among harmonic maps into the sphere, one should single out two particular subclasses, namely the holomorphic and antiholomorphic maps (solutions to $i \partial_{z} u=u \times \partial_{z} u$ and $i \partial_{z} u=-u \times \partial_{z} u$, respectively). In the case where $u$ is holomorphic, $X$ is proportional to $u$, and its image $\Sigma$ is a piece of a sphere. If $u$ is antiholomorphic, then $X$ is minimal. In the remaining cases - the most interesting ones for us $-X$ is a CMC surface whose image does not lie in a sphere. In that latter case, the Ruh-Vilms theorem can be improved on, in the sense that $X$ can be reconstructed from its Gauss map $u$ (up to translations of course). This is achieved through various formulae (Kenmotsu [20], Sym-Bobenko), or using the procedure below. Rewrite equation (8) as a "closure" condition

$$
\partial_{x}\left(u \times \partial_{x} u\right)+\partial_{y}\left(u \times \partial_{y} u\right)=0 .
$$

This implies the existence of a map $B: \Omega \longrightarrow \mathbb{R}^{3}$ such that

$$
\partial_{x} B=u \times \partial_{y} u, \quad \partial_{y} B=-u \times \partial_{x} u .
$$

Then $X=B+u$.

Therefore, in order to build conformal CMC immersions, it suffices to construct harmonic maps (which are neither holomorphic nor antiholomorphic), and this turns out to be easier.

We may analyze harmonic maps into the 2-sphere in an analogous way to that in the previous section. Indeed let $\phi: \Omega \longrightarrow S O(3)$ lift $u$ in the following sense: $\phi(z)=\left(e_{1}(z), e_{2}(z), u(z)\right)$ where $\left(e_{1}(z), e_{2}(z)\right)$ 
is any oriented orthonormal basis of $T_{u(z)} S^{2}$. Its Maurer-Cartan form $\alpha:=\phi^{-1} d \phi$ splits into two pieces $\alpha=\alpha_{0}+\alpha_{1}$ where

$$
\alpha_{0}:=\left\langle d e_{1}, e_{2}\right\rangle\left(\begin{array}{ccc}
0 & -1 & 0 \\
1 & 0 & 0 \\
0 & 0 & 0
\end{array}\right) .
$$

Further, we decompose as above $\alpha_{1}$ in its $(1,0)$ and $(0,1)$ parts $\alpha_{1}^{\prime}+\alpha_{1}^{\prime \prime}$, and set $\alpha_{\lambda}=\lambda^{-1} \alpha_{1}^{\prime}+\alpha_{0}+\lambda \alpha_{1}^{\prime \prime}$ for any $\lambda \in \mathbb{C}^{\star}$. Then $u$ is harmonic if and only if

$$
d \alpha_{\lambda}+\alpha_{\lambda} \wedge \alpha_{\lambda}=0 \quad \forall \lambda \in \mathbb{C}^{\star} .
$$

Reciprocally, the condition (9) on a simply connected domain $\Omega$ ensures the existence for any $\lambda \in \mathbb{C}^{\star}$ of a map $\phi_{\lambda}: \Omega \longrightarrow S O(3)$ such that $d \phi_{\lambda}=\phi_{\lambda} \alpha_{\lambda}$, and consequently of a map $u_{\lambda}$.

This characterization of harmonic maps was obtained by K. Pohlmeyer [29], V. E. Zhakarov, A. B. Shabat [37] and K. Uhlenbeck [35], following a different approach, inspired by the theory of integrable systems.

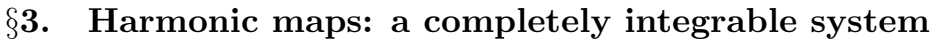

\subsection{Introducing loop groups}

The most efficient way to deal with harmonic maps $u: \Omega \longrightarrow S^{2}$ is to work with the family of maps $\phi_{\lambda}$ constructed above. For the sake of simplicity let us assume (without loss of generality) that $0 \in \Omega$ and $\phi(0)=\mathbb{1}$. From now on $\lambda$ will be a non-zero complex number, unless specified otherwise. Recall from equation (9) the existence of a unique map $\phi_{\lambda}: \Omega \longrightarrow S O(3)$ (for fixed $\lambda$ ) such that

$$
d \phi_{\lambda}=\phi_{\lambda} \alpha_{\lambda} \quad \text { and } \quad \phi_{\lambda}(0)=\mathbb{1} .
$$

Setting

$$
P:=\left(\begin{array}{ccc}
1 & 0 & 0 \\
0 & 1 & 0 \\
0 & 0 & -1
\end{array}\right),
$$

we define a Lie algebra involution by $\tau: \xi \mapsto P \xi P^{-1}$, and observe that

$$
\tau\left(\alpha_{\lambda}\right)=\alpha_{-\lambda} \quad \forall \lambda .
$$


Hence $^{5}$

$$
\tau\left(\phi_{\lambda}\right):=P \phi_{\lambda} P^{-1}=\phi_{-\lambda} .
$$

To verify this we set $\tilde{\phi}_{\lambda}:=\tau\left(\phi_{-\lambda}\right)$. Then $\tilde{\phi}_{\lambda}^{-1} d \tilde{\phi}_{\lambda}=\tau\left(\phi_{-\lambda}^{-1} d \phi_{-\lambda}\right)=$ $\tau\left(\alpha_{-\lambda}\right)=\alpha_{\lambda}$; we conclude the proof by a uniqueness argument.

For this reason we consider the loop group

$$
L S O(3):=\left\{S^{1} \ni \lambda \mapsto g_{\lambda} \in S O(3)\right\},
$$

with group law being pointwise multiplication, and its twisted subgroup

$$
L S O(3)_{\tau}:=\left\{S^{1} \ni \lambda \mapsto g_{\lambda} \in S O(3) \mid \tau\left(g_{\lambda}\right)=g_{-\lambda}\right\} .
$$

The family of maps $\left(\phi_{\lambda}\right)_{\lambda \in S^{2}}$ may therefore be considered as a single map $\phi_{\lambda}: \Omega \longrightarrow L S O(3)_{\tau}$. The construction of harmonic maps from $\Omega$ to $S^{2}$ amounts to finding maps $\phi_{\lambda}: \Omega \longrightarrow L S O(3)_{\tau}$ such that $\phi_{\lambda}^{-1} d \phi_{\lambda}=$ $\lambda^{-1} \alpha_{1}^{\prime}+\alpha_{0}+\lambda \alpha_{1}^{\prime \prime}$.

Remark. The involution $\tau$ gives us a clear explanation for the splitting $\alpha=\alpha_{0}+\alpha_{1}$. Indeed $\tau$ is a Lie algebra automorphism $(\tau([\xi, \eta])=$ $[\tau(\xi), \tau(\eta)])$, which squares to the identity. We may hence decompose the Lie algebra $\mathfrak{s o}(3)$ as the sum of two eigenspaces $\mathfrak{s o}(3)_{0} \oplus \mathfrak{s o}(3)_{1}$ associated respectively to the eigenvalues $(-1)^{0}$ and $(-1)^{1}$. Thus $\alpha_{n}$ is just the projection of $\alpha$ on the $\mathfrak{s o}(3)_{n}$ term.

\subsection{Weierstrass-type representation à la Dorfmeister, Pedit and $\mathrm{Wu}$}

As an application of the formalism introduced above, we describe here an algebraic algorithm for constructing all harmonic maps $\Omega \longrightarrow S^{2}$ (where $\Omega$ is simply connected) starting with holomorphic data. This construction is due to Dorfmeister, Pedit and Wu [9].

Step 1: choosing a potential. Let $a, b: \Omega \longrightarrow \mathbb{C}$ be holomorphic maps, and define a matrix-valued (actually loop algebra-valued) holomorphic 1-form

$$
\mu_{\lambda}=\lambda^{-1}\left(\begin{array}{ccc}
0 & 0 & a \\
0 & 0 & b \\
-a & -b & 0
\end{array}\right) d z
$$

which we call the potential.

\footnotetext{
${ }^{5}$ We use the same letter $\tau$ to denote a group automorphism and its differential at the identity, acting on the Lie algebra.
} 
Step 2: integrating $\mu_{\lambda}$. The potential trivially satisfies $d \mu_{\lambda}+\mu_{\lambda} \wedge$ $\mu_{\lambda}=0$, which is the necessary and sufficient condition for the existence of $g_{\lambda}: \Omega \longrightarrow L S O(3)_{\tau}^{\mathbb{C}}$ such that ${ }^{6} d g_{\lambda}=g_{\lambda} \mu_{\lambda}$.

Step 3: splitting. We write $g_{\lambda}$ as a product $\phi_{\lambda} b_{\lambda}$, with $\phi_{\lambda}: \Omega \rightarrow$ $L S O(3)_{\tau}$ and $b_{\lambda}: \Omega \longrightarrow L^{+} S O(3)_{\tau}^{\mathbb{C}}$, where $L^{+} S O(3)_{\tau}^{\mathbb{C}}$ is the subgroup consisting of loops $b_{\lambda} \in L S O(3)_{\tau}^{\mathbb{C}}$ that are the restriction of holomorphic maps (in $\lambda$ ) from the closed unit disk to $S O(3)^{\mathbb{C}}$.

The map $\phi_{\lambda}$ produced in this way is a lift (in the sense of the previous section) of a harmonic map into the sphere.

A few comments are necessary.

a) The least obvious and most complex operation in the algorithm is the decomposition $g_{\lambda}=\phi_{\lambda} b_{\lambda}$. It rests upon a difficult theorem from A. Pressley and G. Segal [30], stating more precisely that any loop $g_{\lambda} \in$ $L S O(3)^{\mathbb{C}}$ can be written uniquely as the product of $\phi_{\lambda} \in L S O(3)$ and $b_{\lambda} \in L^{+} S O(3)^{\mathbb{C}}$ (hence the decomposition of maps is done pointwise in $z)$. J. Dorfmeister, F. Pedit and H.-Y. Wu call this decomposition the Iwasawa decomposition, for it is an infinite dimensional analog of the classical Iwasawa decomposition (whose prototype is the Gram-Schmidt theorem: any real matrix is the product of an orthogonal matrix by an upper triangular one).

b) This algorithm accounts for the construction of almost all harmonic maps. Actually J. Dorfmeister, F. Pedit and H.-Y. Wu show how to associate to any harmonic map a unique such potential $\mu_{\lambda}$ where the data $(a, b)$ is meromorphic, albeit with non accumulating poles. There are other constructions along the same lines which avoid using meromorphic data (at some cost though).

c) The algorithm parallels the Enneper-Weierstrass representation formula (hence its name). Indeed $\mu_{\lambda}$ is the analog of

$$
f_{\lambda} d z:=\lambda^{-2}\left(\begin{array}{c}
\frac{i}{2}\left(w^{2}-1\right) \\
\frac{1}{2}\left(w^{2}+1\right) \\
i w
\end{array}\right) h d z
$$

${ }^{6} L S O(3)_{\tau}^{\mathbb{C}}$ is the complexification of $L S O(3)_{\tau}$. As a matter of fact $L S O(3)_{\tau}^{\mathbb{C}}:=\left\{S^{1} \ni \lambda \mapsto g_{\lambda} \in S O(3)^{\mathbb{C}} \mid \tau\left(g_{\lambda}\right)=g_{-\lambda}\right\}$ where $S O(3)^{\mathbb{C}}:=$ $\left\{\left.M \in M(3, \mathbb{C})\right|^{t} M M=\mathbb{1}\right.$ and $\left.\operatorname{det} M=1\right\}$. 
The map $g_{\lambda}$ obtained from $\mu_{\lambda}$ corresponds to the (standard) integral $\int^{z} f_{\lambda} d z$ (actually both integrals have the same expression, but the latter takes place in an abelian group). Finally the Iwasawa decomposition reduces to taking the real part. Notice that the analogy is not only in spirit, but that under some conditions, the DPW algorithm reduces to the Enneper-Weierstrass representation formula.

\subsection{Generalizations}

The result of J. Dorfmeister, F. Pedit and H.-Y. Wu applies to any harmonic map from a simply connected $\Omega \subset \mathbb{C}$ to a symmetric space $G / H$, with $G$ compact semisimple. In [15] the first author has built a similar theory for Willmore surfaces. Recall the definition: for any surface $\Sigma$ immersed in $\mathbb{R}^{3}$, we define the Willmore functional as

$$
\mathcal{W}(\Sigma):=\int_{\Sigma} H^{2} d a
$$

where $H$ denotes as usual the mean curvature. The critical points of this functional satisfy the fourth order PDE

$$
\Delta_{\Sigma} H+2 H\left(H^{2}-K\right)=0,
$$

where $K$ is the Gaussian curvature, and they are called Willmore surfaces. Actually this problem had been tackled ten years earlier by K. Voss. Later on it was realized that G. Thomsen had also studied it in 1923 [34], and many important results on these surfaces can be found in W. Blaschke's book [4] (however in 1929, Willmore was only ten years old and Blaschke could not possibly guess that his "conformal minimal surfaces" would become famous under the name of Willmore surfaces). Let us add that S. Germain had already considered the same problem early in the 19th century.

The crucial property of Willmore surfaces is the invariance under conformal transformations of $\mathbb{R}^{3} \cup\{\infty\}$ (also known as the Möbius group of $S^{3}$, which is isomorphic to the connected component of the identity in $S O(4,1))$. This group has dimension 10 , and is generated by the translations, dilations and inversions of $\mathbb{R}^{3}$. Invariance means that for any surface $\Sigma$ without boundary and any Möbius transformation $T$, $\mathcal{W}(T(\Sigma))=\mathcal{W}(\Sigma)$. Consequently the Willmore problem does not rely on the Euclidean structure of $\mathbb{R}^{3}$ but rather on its conformal structure. Since $\mathbb{R}^{3}$ is (locally) conformally equivalent to the sphere $S^{3}$ or the hyperbolic space $H^{3}$, the (local) theory of Willmore surfaces is identical in all three spaces. 
Another important property (rediscovered by R. Bryant [6], though already known to Blaschke) is the existence of the conformal Gauss map, a notion akin to the classical Gauss map of Euclidean geometry, but adapted to conformal geometry. This map takes values in the set $S^{3,1}$ of oriented spheres and planes of $\mathbb{R}^{3}$ (the notation $S^{3,1}$ will be explained below). If $\Sigma$ is an oriented surface in $\mathbb{R}^{3}$, the conformal Gauss map $\gamma$ maps any point $m$ to the unique sphere or plane tangent to $\Sigma$ at $m$ such that their mean curvature vectors coincide - or equivalently with the same orientation and mean curvature. Note that the mean curvature of a sphere is the inverse of its radius; hence the conformal Gauss map is a plane if and only if $H$ vanishes.

A study of conformal geometry shows that the set of oriented spheres and planes of $\mathbb{R}^{3}$ is canonically isomorphic to

$$
\left\{\left.y \in \mathbb{R}^{4,1}|| y\right|^{2}=-\left(y^{0}\right)^{2}+\left(y^{1}\right)^{2}+\left(y^{2}\right)^{2}+\left(y^{3}\right)^{2}+\left(y^{4}\right)^{2}=1\right\}
$$

which is indeed the unit sphere in Minkowski space $\mathbb{R}^{4,1}$, hence the notation. The set $S^{3,1}$ has a natural pseudo-riemannian structure, and is also a symmetric space, being written as $S O(4,1) / S O(3,1)$.

All this construction is motivated by the following analog of the Ruh-Vilms theorem.

Theorem 2 ([4], [6]). Let $X: \Omega \rightarrow \Sigma$ be a conformal parametrization of a surface in $\mathbb{R}^{3}$ or $S^{3}$, and $\gamma: \Omega \rightarrow S^{3,1}$ its conformal Gauss map. Then

- $\gamma$ is weakly conformal, namely for any $z \in \Omega, \gamma$ is conformal at $z$ or $d \gamma_{z}=0$,

- $\quad \Sigma$ is a Willmore surface if and only if $\gamma$ is harmonic.

This suggests immediately a strategy for analyzing and constructing Willmore surfaces, by analogy to the theory of CMC surfaces. One should study harmonic maps from $\Omega$ to $S^{3,1}$ and deduce from them conformal Willmore immersions. However it is trickier to reconstruct the Willmore immersion from the conformal Gauss map than the CMC surface from the classical Gauss map. Although feasible in principle, singularities may arise at umbilic points, hence the difficulties. See [15] for details.

A slightly different strategy proposed in [15] allows us to avoid the problem with the umbilic points. It relies on another kind of Gauss map: for that purpose we choose $\hat{X}: \Omega \longrightarrow S^{3}$ any map such that $X(z) \neq \hat{X}(z), \forall z \in \Omega$. Now there is a canonical way to associate to 
each pair of disjoint points in $S^{3}$ a 3-dimensional spacelike subspace in $\mathbb{R}^{4,1}$. Thus the pair $(X, \hat{X}): \Omega \longrightarrow S^{3} \times S^{3}$ induces a map $Z$ : $\Omega \longrightarrow G r_{3}\left(\mathbb{R}^{4,1}\right)$, where $G r_{3}\left(\mathbb{R}^{4,1}\right)$ is the Grassmannian of 3-dimensional spacelike subspaces contained in $\mathbb{R}^{4,1}$. Of course another choice $\hat{X}^{\prime}$ in place of $\hat{X}$ would lead to another map $Z^{\prime}$ and one goes from $Z$ to $Z^{\prime}$ through a gauge transformation parametrized by a map in $\mathcal{C}^{\infty}\left(\Omega, \mathbb{R}^{2}\right)$. The key point here is that $G r_{3}\left(\mathbb{R}^{4,1}\right)=S O_{0}(4,1) / S O(3) \times S O(1,1)$, so that any map $Z$ can be lifted to a map $\phi: \Omega \longrightarrow S O_{0}(4,1)$; then the Maurer-Cartan form $\omega:=\phi^{-1} d \phi$ can be deformed into a form $\omega_{\lambda}=$ $\lambda^{-1} \omega_{1}^{\prime}+\omega_{0}+\lambda \omega_{1}^{\prime \prime}$, which is curvature-free if and only if $X$ is a conformal Willmore immersion. Therefore we see a way to rely on the results of J. Dorfmeister, F. Pedit and H.-Y. Wu. Note that here $\omega_{1}^{\prime}$ is not a $(1,0)$-form in general, except for special gauge choices of $Z$ (which can be achieved only locally in general).

\section{§4. Another example of integrable system in geometry: Hamil- tonian stationary Lagrangian surfaces}

We will describe now another integrable system which also corresponds to a variational problem in differential geometry, and shares many traits with the problems seen above. Nevertheless this new system cannot be reduced to the study of a harmonic map via some Gauss map, as was the case for CMC or in spirit for Willmore surfaces. Details of this work can be found in $[17,18,19]$.

\subsection{Hamiltonian stationary Lagrangian submanifolds}

A symplectic manifold is a (real) even-dimensional manifold $\mathcal{M}^{2 n}$ endowed with a closed 2 -form $\omega$ that is non-degenerate, i.e. for any point $m \in M$, and any covector $\alpha \in T_{m}^{*} \mathcal{M}$ there exists a unique vector $V \in T_{m} \mathcal{M}$ such that $\omega(V,)=.\alpha$. The form $\omega$ is called a symplectic form. Hereafter we will consider manifolds that are also endowed with a Riemannian metric $g$, compatible with $\omega$ in the sense that the tensor field $J$ defined by $g(J V, W)=\omega(V, W)$ for any $V, W \in T_{m} \mathcal{M}$ is an almost-complex structure, i.e. $J^{2}=-\mathbb{1}$.

The simplest example is $\mathbb{R}^{2 n}$ with the standard scalar product $\langle.,$. and the symplectic form

$$
\omega:=d x^{1} \wedge d y^{1}+\cdots+d x^{n} \wedge d y^{n} .
$$

The corresponding almost-complex structure $J$ is actually complex (meaning that $\mathbb{R}^{2 n} \simeq \mathbb{C}^{n}$ is indeed a complex manifold); it is given in the 
$\left(x^{1}, y^{1}, \ldots, x^{n}, y^{n}\right)$ coordinates by the matrix

$$
\left(\begin{array}{ccccc}
0 & -1 & \ldots & 0 & 0 \\
1 & 0 & \ldots & 0 & 0 \\
\vdots & \vdots & & \vdots & \vdots \\
0 & 0 & \ldots & 0 & -1 \\
0 & 0 & \ldots & 1 & 0
\end{array}\right)
$$

The metric and symplectic structures merge into the standard Hermitian product $\langle., .\rangle_{H}:=\langle.,\rangle-.i \omega(.,$.$) . As a consequence, the group of$ linear transformations of $\mathbb{R}^{2 n}$ preserving both the scalar product and the symplectic form is $U(n)$, the group which preserves the Hermitian product.

We now mix both structures to set up a variational problem. Using the metric $g$, one defines the volume of any immersed submanifold $\Sigma$ of $\mathcal{M}, \mathcal{A}(\Sigma):=\int_{\Sigma} d \mathrm{Vol}$. We study critical points of this functional but restricted to (i) Lagrangian submanifolds $\Sigma$ and (ii) Hamiltonian variations (which in particular preserve the condition of being Lagrangian). A Lagrangian submanifold of $(\mathcal{M}, \omega)$ is a submanifold $\Sigma$ of dimension $n$ such that the restriction of $\omega$ to $\Sigma$ vanishes: $\omega_{\mid \Sigma}=0$. Equivalently, using the almost-complex structures, $J T_{m} \mathcal{M}$ is orthogonal to $T_{m} \mathcal{M}$ for any $m \in \mathcal{M}$. A vector field $V$ on $\mathcal{M}$ is said to be Hamiltonian if there exists $h \in C^{\infty}(\mathcal{M}, \mathbb{R})$ such that $d h()=.\omega(V,$.$) (in other words V=-J \nabla h$ ). We write then $V=\xi_{h}$ and call $V$ the symplectic gradient of $h$. It is easy to show that the flow of a Hamiltonian vector field preserves Lagrangian submanifolds, i.e. the image of a Lagrangian submanifold by the flow is again a Lagrangian submanifold.

A Hamiltonian stationary Lagrangian submanifold is a Lagrangian submanifold such that the volume is constant up to first order for any Hamiltonian variation: for any $h \in \mathcal{C}^{\infty}(\mathcal{M}, \mathbb{R}), \delta \mathcal{A}\left(\xi_{h}\right)=0$. In general this notion is weaker than minimality (for a Lagrangian submanifold). Indeed let $\mathcal{H}$ be the mean curvature vector. By definition it is the unique normal vector field along $\Sigma$ such that for any infinitesimal variation $V$ of $\Sigma$,

$$
\delta \mathcal{A}(\Sigma)(V)=\int_{\Sigma} g(\mathcal{H}, V) d V o l
$$

Then a Hamiltonian stationary Lagrangian submanifold is a solution to the following system

$$
\left\{\begin{aligned}
\omega_{\mid \Sigma} & =0 \\
\int_{\Sigma} g(J \mathcal{H}, \nabla h) d V o l & =0, \quad \text { for any } h \in \mathcal{C}^{\infty}(\mathcal{M}, \mathbb{R})
\end{aligned}\right.
$$




\section{Examples}

a) In $\mathbb{R}^{2}$ the symplectic form is just the volume form $d x \wedge d y$. For dimensional reasons, any curve is Lagrangian. The minimal curves are the straight lines, but Hamiltonian stationary curves are the critical points of length for area-preserving variations. Thus these are straight lines and circles. In this case the problem of Hamiltonian stationary Lagrangian submanifolds is akin to the isoperimetric problem.

b) In $\mathbb{R}^{4}$ an example of Hamiltonian stationary Lagrangian surface is the flat torus

$$
S^{1} \times S^{1}=\left\{\left(x^{1}, y^{1}, x^{2}, y^{2}\right) \in \mathbb{R}^{4} \mid\left(x^{1}\right)^{2}+\left(y^{1}\right)^{2}=\left(x^{2}\right)^{2}+\left(y^{2}\right)^{2}=\frac{1}{2}\right\} .
$$

In [27], Y. G. Oh has conjectured that this torus minimizes area among all tori obtained from it through Hamiltonian deformations. Under regularity assumptions, a proof of that conjecture has been obtained by $\mathrm{H}$. Anciaux [2].

\subsection{Characterizing Hamiltonian stationary submanifolds}

In order to understand further the geometry of these submanifolds, we need a better formulation than (12) for the variational problem, and in particular for the mean curvature vector.

Considering first the case $\mathcal{M}=\mathbb{R}^{2 n} \simeq \mathbb{C}^{n}$, let us tackle the problem from the Ruh-Vilms theorem angle and connect it to the Gauss map of a Lagrangian immersion. Obviously this map takes values in the Grassmannian of oriented Lagrangian $n$-planes

$$
G r_{L a g}\left(\mathbb{C}^{n}\right) \simeq U(n) / S O(n) \simeq U(1) \times(S U(n) / S O(n)) .
$$

The Gauss map we will consider is the map $\gamma: \Sigma \rightarrow G r_{\text {Lag }}\left(\mathbb{C}^{n}\right)$ sending each $m \in \Sigma$ to its oriented tangent space $\gamma(m)=T_{m} \Sigma$. As mentioned above, $G r_{L a g}$ splits into two parts and we can write $\gamma=\left(e^{i \beta}, \tilde{\gamma}\right)$ where $e^{i \beta}$ (respectively, $\tilde{\gamma}$ ) takes values in $U(1)$ (respectively, $S U(n) / S O(n)$ ). The $\mathbb{R} / 2 \pi \mathbb{Z}$-valued map $\beta$ is called the Lagrangian angle and we claim it is the only relevant part of the Gauss map, as far as Hamiltonian stationary submanifolds are concerned.

Lemma 1. Let $\Sigma$ be a Lagrangian submanifold. Then the mean curvature vector of $\Sigma$ is

$$
\mathcal{H}=J \nabla \beta,
$$

where $e^{i \beta}$ is the $U(1)$ component of the Gauss map. 
Note that, even if $\beta$ is only defined modulo $2 \pi$, its gradient is welldefined everywhere. Finally we apply lemma 1 to equation (12) to conclude that

$$
\int_{\Sigma} g(J H, \nabla h) d V o l=-\int_{\Sigma} g(\nabla \beta, \nabla h) d V o l=\int_{\Sigma} h \Delta_{\Sigma} \beta d V o l .
$$

Hence:

Theorem 3. A Lagrangian submanifold $\Sigma$ of $\mathbb{C}^{n}$ is Hamiltonian stationary if and only if

$$
\Delta_{\Sigma} \beta=0
$$

Despite its compact form, this equation is a third order nonlinear PDE. Indeed the operator $\Delta_{\Sigma}$ depends (nonlinearly) on the immersion $X$, and $\beta$ depends on the first order derivatives of $X$.

One may wonder how to extract painlessly the angle $\beta$. Simply pick any oriented orthonormal frame $\left(e_{1}, \ldots, e_{n}\right)$ of $T_{m} \Sigma$; then

$$
e^{i \beta}=\theta\left(e_{1}, \ldots, e_{n}\right) \text { where } \theta=d z^{1} \wedge \ldots \wedge d z^{n}
$$

This characterization offers a seamless generalization to Calabi-AubinYau manifolds. These manifolds are complex Kähler manifolds (i.e. the almost-complex structure is complex and parallel) with flat Ricci tensor. As a crucial consequence, the canonical bundle is flat. Recall that the canonical bundle of a complex manifold is the (complex) one-dimensional bundle $K$ of $(n, 0)$-forms (locally generated by $\left.d z^{1} \wedge \ldots \wedge d z^{n}\right)$. The metric induces a connection on $K$ whose curvature is a multiple of the Ricci form $\rho=\operatorname{Ric}(J .,$.$) ; hence the flatness of K$. Consequently there exists a (local) nonzero parallel section $\theta$ of $K$, which generalizes $d z^{1} \wedge \ldots \wedge d z^{n}$, and we define the Lagrangian angle in the same fashion.

The definition of Lagrangian angle along a Lagrangian submanifold can also be extended to Kähler-Einstein manifolds (see [36]). In that case the Ricci tensor is a multiple of the metric, and consequently the Ricci form is proportional to the symplectic form $\omega$. Thus the curvature of the canonical bundle vanishes along a Lagrangian submanifold. We can define a parallel section $\theta$ along $\Sigma$ only (not on the whole manifold) and give meaning to the Lagrangian angle. Then again $\mathcal{H}=J \nabla \beta$.

\subsection{Hamiltonian stationary Lagrangian surfaces in $\mathbb{R}^{4}$}

We specialize now to Lagrangian surfaces in $\mathbb{R}^{4} \simeq \mathbb{C}^{2}$. In this case we may assume without loss of generality that the surface is given by a 
conformal immersion $X: \Omega \rightarrow \mathbb{R}^{4}$. A usual we take $\Omega$ to be a simply connected domain in the plane.

Inspired by the previous constructions for CMC surfaces, we build a moving frame $\left(e_{1}, e_{2}\right)$ in $\mathbb{R}^{4}$ such that

$$
\left\{\begin{array}{ccc}
\left\langle e_{a}(z), e_{b}(z)\right\rangle & = & \delta_{a b} \\
\omega\left(e_{1}(z), e_{2}(z)\right) & = & 0
\end{array} \Longleftrightarrow\left\langle e_{a}(z), e_{b}(z)\right\rangle_{H}=\delta_{a b}\right.
$$

for any $z \in \Omega$. In other words $\left(e_{1}, e_{2}\right)$ is an unitary basis of $\mathbb{C}^{2}$ over $\mathbb{C}$, or $\left(e_{1}, e_{2}\right)$ are the columns of a matrix in $U(2)$. That way we have constructed a map $F: \Omega \rightarrow U(2) \ltimes \mathbb{C}^{2}$ lifting $X$, defined by

$$
F(z):=\left(\begin{array}{ccc}
e_{1}(z) & e_{2}(z) & X(z) \\
0 & 0 & 1
\end{array}\right) .
$$

Before going further we need to understand what we are looking for. If we lift $X$ into $U(2) \ltimes \mathbb{C}^{2}$ (using Darboux frames for instance), the lift $F$ will include first order information on $X$ and the Euler-Lagrange equation (13) will be reduced to second order (as in the harmonic map case). If we hope to go one step further, it is judicious to lift the pair $(X, \gamma)$ - which takes values in $\left(U(2) \ltimes \mathbb{C}^{2}\right) / S O(2)$ - where $\gamma$ is the Gauss map defined above. Since $(X, \gamma)$ contains first order data (on $X$ ), the lift should contain second order derivatives. Such a solution is feasible but hardly optimal; indeed the only relevant part of the Gauss map is the Lagrangian angle. So we want to lift the pair $\left(X, e^{i \beta}\right)$, which is a $U(2) \ltimes \mathbb{C}^{2} / S U(2)$ map.

To achieve this, we require from the lift $F$ (defined as above) that

$$
\theta\left(e_{1}, e_{2}\right)=e^{i \beta} \text { where } \beta \text { is the Lagrangian angle along } \Sigma \text {. }
$$

From now on, the lift will always be such (and we call it a Lagrangian lift). Let $\alpha:=F^{-1} d F$ be the Maurer-Cartan form, taking values in the Lie algebra of $U(2) \ltimes \mathbb{C}^{2}, \mathfrak{g}=\mathfrak{u}(2) \oplus \mathbb{C}^{2}$. To have a better understanding of the geometry of this Lie algebra, we introduce the matrix

$$
K:=\left(\begin{array}{ccccc}
0 & 0 & -1 & 0 & 0 \\
0 & 0 & 0 & 1 & 0 \\
1 & 0 & 0 & 0 & 0 \\
0 & -1 & 0 & 0 & 0 \\
0 & 0 & 0 & 0 & 1
\end{array}\right),
$$

(recall that although we write $U(2)$ we always think of real matrices), and define an automorphism $\tau$ on $U(2) \ltimes \mathbb{C}^{2}$ by $g \mapsto K g K^{-1}$. Abusing 
notation we write $\tau$ again for its differential at identity, acting linearly on $\mathfrak{g}$. Since $\tau^{4}=i d$, its action diagonalizes on $\mathfrak{g}^{\mathbb{C}}=\left(\mathfrak{u}(2) \oplus \mathbb{C}^{2}\right)^{\mathbb{C}}$ which splits into four eigenspaces $\mathfrak{g}_{\ell}^{\mathbb{C}}$ for each eigenvalue $i^{\ell}$, where $\ell \in\{-1,0,1,2\}$. So we may write

$$
\alpha=\alpha_{-1}+\alpha_{0}+\alpha_{1}+\alpha_{2} .
$$

As a direct consequence of the choice of lift, each component has a geometrical meaning: $\alpha_{ \pm 1}$ contain $\left\langle d X, e_{a}\right\rangle, \alpha_{0}$ contains the $\mathfrak{s u}(2)$ part, and $\alpha_{2}$ is more or less $d \beta$, indeed this is the second order information we aimed at. As in the harmonic map case we can split into $d z$ and $d \bar{z}$ parts, writing $\alpha_{\ell}=\alpha_{\ell}^{\prime}+\alpha_{\ell}^{\prime \prime}$. We read off the splitting all the geometrical properties we need.

Theorem 4. a) The Lagrangian immersion $X$ is conformal if and only if $\alpha_{1}^{\prime}=\alpha_{-1}^{\prime \prime}=0$.

b) Let $X$ be a conformal Lagrangian immersion, then $X$ is Hamiltonian stationary if and only if

$$
d \alpha_{\lambda}+\alpha_{\lambda} \wedge \alpha_{\lambda}=0, \quad \text { for any } \lambda \in S^{1} \subset \mathbb{C},
$$

where

$$
\alpha_{\lambda}:=\lambda^{-2} \alpha_{2}^{\prime}+\lambda^{-1} \alpha_{-1}+\alpha_{0}+\lambda \alpha_{1}+\lambda^{2} \alpha_{2}^{\prime \prime} .
$$

Equation (15) puts forward a family of deformations of $\alpha=F^{-1} d F$ in a manner similar to what happens in the CMC or harmonic case. In particular, (15) is the necessary and sufficient condition for the existence of $F_{\lambda}: \Omega \rightarrow U(2) \ltimes \mathbb{C}^{2}$ such that $d F_{\lambda}=F_{\lambda} \alpha_{\lambda}$; and each $F_{\lambda}$ yields by projection on $\mathbb{C}^{2}$ a conformal Hamiltonian stationary Lagrangian immersion $X_{\lambda}$.

This result shows that the Hamiltonian stationary problem is an integrable system. We may at that point use appropriate tools like the twisted loop groups

$$
L\left(U(2) \ltimes \mathbb{C}^{2}\right)_{\tau}:=\left\{S^{1} \ni \lambda \mapsto g_{\lambda} \in U(2) \ltimes \mathbb{C}^{2} \mid \tau\left(g_{\lambda}\right)=g_{i \lambda}\right\},
$$

and obtain essentially the same results as for CMC surfaces: a Weierstrasstype formula and a classification of all tori (which are of finite type again).

\subsection{Generalization to other symmetric spaces}

Switching from flat $\mathbb{R}^{4}$ to other Hermitian symmetric spaces of dimension $2\left(\mathbb{C} P^{2}, \mathbb{C} P^{1} \times \mathbb{C} P^{1}\right.$ and their non-compact duals), it remains 
true that Hamiltonian stationary Lagrangian surfaces are the solutions of an integrable system, even if the Lagrangian angle is not anymore globally defined (since these manifolds are not Calabi-Aubin-Yau) [19]. Nevertheless the Lagrangian angle can be defined along the surface only, because the manifolds are Kähler-Einstein. Furthermore, the integrable system retains the same structure; the only change lies in the Lie algebra $\mathfrak{g}$ in which the Maurer-Cartan form takes values. For instance, we write the complex projective plane as $\mathbb{C} P^{2}=S U(3) / U(2)$ and $\mathfrak{g}=\mathfrak{s u}(3)$. As above, we have a notion of an "appropriate" lift of $\left(X, e^{i \beta}\right)$, and also an order four automorphism with the same geometric properties. All results hold, up to the loop group splittings which depend on the compactness of $\mathfrak{g}$; at that point we have either global or local results, according to the space under consideration.

The case of Lagrangian surfaces in $\mathbb{C} P^{2}$ deserves special attention due to its relation with conical singularities of Lagrangian surfaces in complex 3-manifolds. Indeed such singularities have a limit cone $C \subset$ $\mathbb{C}^{3}$ whose intersection $\hat{\Sigma}$ with the unit sphere is a Legendrian surface, namely the tangent plane at any $m \in \hat{\Sigma} \subset S^{5}$ is orthogonal to both $m$ and $\mathrm{im}$. This is a contact condition: $\hat{\Sigma}$ is tangent to a four dimensional non integrable distribution in $S^{5}$. The Hopf fibration $\pi: S^{5} \rightarrow \mathbb{C} P^{2}$ projects Legendrian surfaces down to Lagrangian surfaces. Conversely, any (simply connected) Lagrangian surface admits a unique Legendrian lift through $\pi$, up to a multiplicative constant, giving rise to a Lagrangian cone in $\mathbb{C}^{3}$. Moreover Hamiltonian stationarity is preserved from the cone to the projected surface in $\mathbb{C} P^{2}$. So is minimality, so that specialLagrangian cones in $\mathbb{C}^{3}$ correspond to minimal Lagrangian surfaces in $\mathbb{C} P^{2}$, a subcase of our integrable system. Notice that an intermediate integrable system can be written for Legendrian surfaces in $S^{5}$.

\subsection{Another approach for Lagrangian surfaces in $\mathbb{R}^{4}$}

Finally let us inspect more carefully Hamiltonian stationary Lagrangian surfaces in $\mathbb{R}^{4}$. Using the splitting $\mathfrak{g}^{\mathbb{C}}=\mathfrak{g}_{-1}^{\mathbb{C}}+\mathfrak{g}_{0}^{\mathbb{C}}+\mathfrak{g}_{1}^{\mathbb{C}}+\mathfrak{g}_{2}^{\mathbb{C}}$, equation (15) uncouples to yield (almost) linear systems. It turns out that constructing such surfaces amounts to solving consecutively two linear PDEs, and integrating, a procedure much simpler than the infinite dimensional methods described previously. Even more, this process applies actually to Lagrangian surfaces themselves, not only the stationary ones. Before we proceed with the construction, let us point out its limitations: since the decoupling relies upon commutation properties, it 
will never apply to other Hermitian symmetric spaces like $\mathbb{C} P^{2}$, nor to the CMC case.

The simplest way to obtain these results consists in taking a particular lift $F$ among the Lagrangian lifts of $\left(X, e^{i \beta}\right)(X$ is as usual a conformal Lagrangian immersion, not necessarily stationary). Indeed, choose $F=\left(e^{i \beta} \epsilon_{1}, e^{i \beta} \epsilon_{2}, X\right)$ or $\left(-e^{i \beta} \epsilon_{1},-e^{i \beta} \epsilon_{2}, X\right)$, where $\left(\epsilon_{1}, \epsilon_{2}\right)$ is any unitary basis. Clearly $F$ satisfies our axiom from section 4.3. We may actually write

$$
e^{-J \beta / 2} d X=\operatorname{Re}\left[\left(\begin{array}{c}
s_{1} \\
-i s_{1}
\end{array}\right) d z\right]+i \operatorname{Re}\left[\left(\begin{array}{c}
s_{2} \\
i s_{2}
\end{array}\right) d z\right]
$$

where $s_{1}$ and $s_{2}$ are complex-valued functions. If we set

$$
\phi:=\left(\begin{array}{c}
s_{1} \\
\bar{s}_{2}
\end{array}\right),
$$

then $\phi$ satisfies the Dirac-type equation

$$
\mathcal{D} \phi=M \phi,
$$

where

$$
\mathcal{D}:=\left(\begin{array}{cc}
0 & \partial / \partial z \\
-\partial / \partial \bar{z} & 0
\end{array}\right) \text { and } \quad M:=\frac{1}{2}\left(\begin{array}{cc}
\partial \beta / \partial \bar{z} & 0 \\
0 & \partial \beta / \partial z
\end{array}\right) .
$$

Reciprocally, given any map $\beta: \Omega \rightarrow \mathbb{R}$ and any $\phi$ solution of (16), then the $\mathbb{C}^{2}$-valued one-form

$$
\xi:=e^{J \beta / 2} \operatorname{Re}\left(\left(\begin{array}{c}
s_{1} \\
-i s_{1}
\end{array}\right) d z\right)+i e^{J \beta / 2} \operatorname{Re}\left(\left(\begin{array}{c}
s_{2} \\
i s_{2}
\end{array}\right) d z\right)
$$

is closed: $d \xi=0$. Thus there is a map $X: \Omega \rightarrow \mathbb{C}^{2} \simeq \mathbb{R}^{4}$ such that $d X=\xi$ and whenever $\phi$ does not vanish, $X$ is a conformal Lagrangian immersion. Furthermore, $X$ is Hamiltonian stationary if and only if $\beta$ is harmonic.

An alternative approach was developped by R. Aiyama [1]. We explain here the connection with our framework. Write

$$
X=: e^{i \beta / 2}\left(\begin{array}{c}
a+b \\
i(\bar{a}-\bar{b})
\end{array}\right), \text { and } \Phi:=\left(\begin{array}{c}
a+\bar{b} \\
i(\bar{a}-b)
\end{array}\right) .
$$

Clearly the components of the immersion $X$ can be expressed in terms of $\Phi$ and $\beta$ without integration. But $\Phi$ satisfies also a Dirac equation: 
$\mathcal{D} \Phi=-\bar{M} \Phi$. The variables $\Phi$ and $\phi$ are related by

$$
\phi=(\mathcal{D}+\bar{M})\left(\begin{array}{cc}
0 & -1 \\
1 & 0
\end{array}\right) \Phi .
$$

We obtain in this way a Weierstrass representation of sorts for Lagrangian surfaces in $\mathbb{R}^{4}$. Analogous formulations have already been suggested for surfaces in $\mathbb{R}^{3}$, extending the Enneper-Weierstrass formula to the non-minimal case; see for instance the work of B. G. Konopelchenko [21]. It is quite instructive to compare his representation formula with the one for Lagrangian surfaces we have just obtained. The representation formula of B. G. Konopelchenko consists in choosing a real-valued function $p$ on $\Omega$, called the potential, and a map $\phi: \Omega \rightarrow \mathbb{C}^{2}$ (actually a spinor) such that $\mathcal{D} \phi=p \phi$ (with the same notation as before). Then the $\mathbb{R}^{3}$ valued one-form

$$
\eta:=\operatorname{Re}\left(\begin{array}{c}
s_{1}^{2} d z-{\overline{s_{2}}}^{2} d \bar{z} \\
-i\left(s_{1}^{2} d z-{\overline{s_{2}}}^{2}\right) d \bar{z} \\
s_{1} s_{2} d z+\overline{s_{1} s_{2}} d \bar{z}
\end{array}\right)
$$

satisfies $d \eta=0$. Hence the existence of a map $Y: \Omega \rightarrow \mathbb{R}^{3}$ such that $d Y=\eta$; furthermore $Y$ is a weakly-conformal immersion with first fundamental form

$$
I=\left(\left|s_{1}\right|^{2}+\left|s_{2}\right|^{2}\right)^{2}\left(\begin{array}{ll}
1 & 0 \\
0 & 1
\end{array}\right)
$$

and mean curvature given by $2 p=H\left(\left|s_{1}\right|^{2}+\left|s_{2}\right|^{2}\right)$.

However both formulae differ deep inside in that the quantities involved $(\phi, \beta, p)$ are of a different nature in each problem (e.g. $p$ is a density while $\beta$ is a function), and also because one is quadratic while the other is essentially linear. Still there are interesting analogies and in some particular cases, both Dirac equations coincide. Thus some Lagrangian surfaces in $\mathbb{R}^{4}$ correspond to some surfaces in $\mathbb{R}^{3}$.

This Dirac-like approach yields quite simple expressions in the Hamiltonian stationary case. Notably it leads to a complete description of all such tori in $\mathbb{R}^{4}$. Using this representation, H. Anciaux [2] has obtained precise isoperimetric estimates in relation to Oh's conjecture.

\section{$\S 5$. Conclusion}

In this survey, we have exhibited only some aspects of integrable systems theory, and skipped interesting developments such as finite type 
solutions (e.g. all CMC tori have finite type, as shown by U. Pinkall and I. Sterling [28] (see also [16])), the dressing action, and so on. We have also given few examples. For more details of the general theory see $[13,33,16]$; for Hamiltonian stationary Lagrangian surfaces, see $[17,18,19]$.

\section{References}

[1] R. Aiyama, Totally real surfaces in the complex 2-space, Steps in Differential Geometry, Proceedings of the Colloquium on Differential Geometry, 25-30 July, 2000, Debrecen, Hungary, http://pc121.math.klke.hu/diffgeo/ and Lagrangian surfaces in the complex 2-space, Proceedings of the 5th International Workshops on Differential Geometry, Kyungpook National University, Korea, 2000.

[2] H. Anciaux, An isoperimetric inequality for Hamiltonian stationary Lagrangian tori in $\mathbb{C}^{2}$ related to Oh's conjecture, Math. Z. 241 (2002), 639-664

[3] L. Bianchi, Lezioni di geometria differenziale, Spoerri, Pisa, 1902.

[ 4 ] W. Blaschke, Vorlesungen über Differentialgeometrie III, Springer Berlin 1929.

[5] O. Bonnet, Note sur une propriété de maximum relative à la sphère, Nouvelles Annales de Mathématiques, t. XII (1853), 433.

[6] R. Bryant, A duality theorem for Willmore surfaces, J. Differential Geometry 20 (1984), 23-53. Surfaces in conformal geometry, Proc. Symp. Pure Math. Amer. Math. Soc. 48 (1988), 227-240.

[7] G. Darboux, Leçons sur la théorie générale des surfaces, livre VII, Chapitre X, Gauthiers-Villars 1884.

[8] H. Dobriner, Über die Flächen mit einem System sphärischer Krümmungslinien, J. reine angew. Math. (Crelle's Journal) 94 (1883), 116-161. Die Flächen Constanter Krümmung mit einem System Sphärischer Krümmungslinien dargestellt mit Hilfe von Theta Functionen Zweier Variabeln, Acta Math. 9 (1886), 73-104.

[9] J. Dorfmeister, F. Pedit and H.Y. Wu, Weierstrass type representation of harmonic maps into symmetric spaces, Comm. in Analysis and Geom. 6 (1998), 633-668.

[10] A. Enneper, Analytisch-geometrische Untersuchungen, Göttingen Nachr. (1868), 258-277 and 421-443. Untersuchungen über die Flächen mit planen und sphärischen Krümmungslinien, Abh. Königl. Ges. Wissensch. Göttingen 23 (1878) and 26 (1880). Über die Flächen mit einem System sphärischen Krümmungslinien, J. reine angew. Math. (Crelle's Journal) 94 (1883), 329-341. 
[11] A. Fordy, J.C. Wood, Harmonic maps and integrable systems, Aspects of Mathematics E23, Vieweg 1994 (see contents at www.amsta.leeds.ac.uk/Pure/staff/wood/FordyWood/contents.html).

[12] C. Gardner, J. Greene, M. Kruskal and R. Miura, Method for solving the Korteweg-de Vries equation, Phys. Rev. Lett. 19 (1967), 1095-1097.

[13] M. Guest, Harmonic maps, loop groups and integrable systems, Cambridge University Press, Cambridge 1997.

[14] F. Hélein, Applications harmoniques, lois de conservation et repères mobiles, Diderot éditeur, 1996, or Harmonic maps , integrable systems and moving frames, Diderot éditeur, 1997.

[15] F. Hélein, Willmore immersions and loop groups, J. Differential Geometry 50 (1998), 331-385. See also Weierstrass representation for Willmore surfaces, in Harmonic morphisms, harmonic maps and related topics, C.K. Anand, P. Baird, E. Loubeau and J.C. Wood editors, Chapman and Hall/CRC Research Notes in Mathematics 413.

[16] F. Hélein, Constant mean curvature surfaces, harmonic maps and integrable systems, Lectures in Mathematics, ETH Zürich, Birkhäuser 2001.

[17] F. Hélein, P. Romon, Hamiltonian stationary Lagrangian surfaces in $\mathbb{C}^{2}$, Commun. Anal. Geom. 10 (2002), 79-126.

[18] F. Hélein, P. Romon, Weierstrass representation of Lagrangian surfaces in four-dimensional space using spinors and quaternions, Comment. Math. Helv. 75 (2000), 668-680.

[19] F. Hélein, P. Romon, Hamiltonian stationary Lagrangian surfaces in Hermitian symmetric spaces, Differential geometry and integrable systems, Contemp. Math. 308 (2002), 161-178.

[20] K. Kenmotsu, Weierstrass formula for surfaces of prescribed mean curvature, Math. Ann. 245 (1979), 89-99.

[21] B. G. Konopelchenko, Induced surfaces and their integrable dynamics, Studies in Appl. Math. 96, No.1, 9-51 (1996). See also: B. G. Konopelchenko, I. Taimanov, Constant curvature surfaces via integrable dynamical system, J. Phys. A, Math. Gen. 29, No. 6, 1261-2165 (1996) et B. G. Konopelchenko, I. Taimanov, Generalized Weierstrass formulae, soliton equations and Willmore surfaces I. Tori of revolution and the $m K d V$ equation, preprint.

[22] T. Kuen, Flächen von constanten negativem Krümmungsmass nach L. Bianchi, Sitzungsber. der Bayer. Akad. (1884).

[23] P.D. Lax, Integrals of nonlinear equations of evolution and solitary waves, Comm. Pure. Appl. Math. 21 (1968), 467-490.

[24] E. Lenz, Über Enneperschen Flächen konstanten negativen Krümmungsmasses, Dissertation, Universität Göttingen, 1879.

[25] S. Lie, Zur Theorie des Flächen konstanter Krümmung, Archiv for Mathematik og Naturvidenskab (I u. II) (1879) and (III) (1880), Kristiania.

[26] M. Melko, I. Sterling, Integrable systems, harmonic maps and the classical theory of solitons, in [11]. 
[27] Y.G. Oh, Second variation and stabilities of minimal Lagrangian submanifolds in Kähler manifolds, Invent. Math. 101 (1990), 501-519. Volume minimization of Lagrangian submanifolds under Hamiltonian deformations, Math. Z. 212 (1993), 175-192.

[28] U. Pinkall, I. Sterling, On the classification of constant mean curvature tori, Annals of Math. 130 (1989), 407-451.

[29] K. Pohlmeyer, Integrable Hamiltonian systems and interactions through constraints, Comm. Math. Phys. 46 (1976), 207-221.

[30] A. Pressley and G. Segal, Loop groups, Oxford Mathematical Monographs, Clarendon Press, Oxford 1986.

[31] E. Ruh, J. Vilms, The tension field of the Gauss map, Trans. Amer. Math. Soc. 149 (1970), 569-573.

[32] W. Symes, Systems of Toda type, inverse spectral problems and representation theory, Invent. Math. 159 (1980), 13-51.

[33] C.L. Terng, K. Uhlenbeck, Integrable systems, J. Differential Geometry Surveys, vol. 4, International Press, Cambridge, MA, 1999.

[34] G. Thomsen, Über konforme Geometrie I: Grundlagen der konformen Flächentheoremrie, Abh. Math. Sem. Hamburg (1923), 31-56.

[35] K. Uhlenbeck, Harmonic maps into Lie groups, Journal of Differential Geometry 30 (1989), 1-50.

[36] J. Wolfson, Minimal Lagrangian diffeomorphisms and the Monge-Ampère equations, J. Differential Geometry 46 (1997), 335-373.

[37] V.E. Zhakarov, A.B. Shabat, Integration of nonlinear equations of mathematical physics by the method of inverse scattering, Funktsional'nyi Analiz i Ego Prilozheniya, Vol. 13, No. 3, 13-22, July-September 1979.

\author{
Frédéric Hélein \\ CMLA, ENS de Cachan \\ 61 avenue du Président Wilson \\ 94235 Cachan Cedex \\ FRANCE \\ Pascal Romon \\ Université de Marne-la-Vallée \\ 5 bd Descartes \\ Champs-sur-Marne \\ 77454 Marne-la-Vallée Cedex 2 \\ FRANCE \\ E-mail address: \\ helein@math.jussieu.fr \\ $\operatorname{xxxxxxxxxxxxxxx}$
}

